\title{
Delayed presentation of psoas abscess previously misdiagnosed as deep venous thrombosis: A potentially devastating error
}

\author{
Victor Kong, George Oosthuizen, Musa Mthethwa, Kriban Reddy, \\ Damian Clarke
}

\begin{abstract}
Introduction: Psoas abscess is an uncommon condition seen only occasionally in daily surgical practice. Often, its clinical presentation is non-specific, frequently causing diagnostic uncertainty. Late presentation, delayed recognition and inappropriate management of this condition can often precede eventual diagnosis, especially in resource poor settings. Case Report: We describe the case of a 22-yearold man who presented with a large psoas abscess which unfortunately had been misdiagnosed and treated as a deep venous thrombosis. Conclusion: This unusual case highlights the difficulties often encountered by clinicians when assessing and managing a patient with a psoas abscess. The diagnosis of a psoas abscess remains challenging. Clinicians must always remain vigilant to this diagnosis. Early recognition, confirmation with imaging and swift drainage remains the key to optimising patient's outcome.
\end{abstract}

Victor Kong ${ }^{1}$, George Oosthuizen², Musa Mthethwa ${ }^{3}$, Kriban Reddy $^{3}$, Damian Clarke ${ }^{2}$

Affiliations: 1Surgical Registrar, Department of Surgery, Edendale Hospital, Pietermaritzburg, KwaZulu Natal, South Africa; ${ }^{2}$ Senior Consultant Surgeon, Department of Surgery, Edendale Hospital, Pietermaritzburg, KwaZulu Natal, South Africa; ${ }^{3}$ Consultant Surgeon, Department of Surgery, Edendale Hospital, Pietermaritzburg, KwaZulu Natal, South Africa.

Corresponding Author: Victor Yeewai Kong, Department of Surgery, Edendale Hospital, Private Bag X509, Plessislaer, Pietermaritzburg, 3216. KwaZulu Natal, South Africa; Cell: +27(0)797411036; Fax: +27(0)333954094; Email: victorywkong@yahoo.com

Received: 27 July 2012

Accepted: 18 October 2012

Published: 01 February 2013
Keywords: Psoas, Abscess, Diagnosis, Error

$$
* * * * * * * * *
$$

Kong V, Oosthuizen G, Mthethwa M, Reddy K, Clarke D. Delayed presentation of psoas abscess previously misdiagnosed as deep venous thrombosis: A potentially devastating error. International Journal of Case Reports and Images 2013;4(2):111-114.

$* * * * * * * * *$

doi:10.5348/ijcri-2013-02-273-CR-7

\section{INTRODUCTION}

Psoas abscesses are rare. Clinical diagnosis is extremely difficult because presentation is often subtle and non-specific. This case report focuses on a patient who presented to our surgical unit with a large psoas abscess after the condition had been wrongly diagnosed and mistreated as deep venous thrombosis at another rural hospital.

\section{CASE REPORT}

The patient was a 22-year-old male with no previous known medical history. He presented to another rural hospital with complaints of gradual onset of a vague discomfort in his right hip. He also noted a slight swelling of his right foot and reduced mobility of the right hip, which remained unchanged after weight bearing. He had no previous history of a similar presentation and no recent history of trauma. The patient's HIV status was unknown at the time and he reported no previous tuberculosis contact. He had been treated by a non-specialist doctor at another rural hospital who diagnosed a deep venous thrombosis (however, an ultrasound Doppler facility was not 
available at this hospital) and the patient was commenced on therapeutic dose of subcutaneous enoxaparin, $40 \mathrm{mg}$ daily for one week. The patient did not experience any improvement and continued to complain of an increasing pain in the affected side. Subsequently, he did not attend his follow up appointment at his local hospital due to the lack of available transportation. However, after two weeks, he managed to return to the hospital and was eventually referred to our surgical unit for a second opinion.

On examination, the patient appeared emaciated and was in obvious discomfort. He was apyrexial, with a pulse of 100/min and a blood pressure of 135/65 $\mathrm{mmHg}$. An ill defined tenderness in the right iliac fossa was noted, extending along the inguinal ligament, with no palpable mass. A slightly swollen right foot with the swelling extending to the calf was also noted (Figure 1). The range of motion in his right hip was slightly reduced. His leukocyte count was $1.8 \times 10^{4} / \mathrm{mm}^{3}$. His remaining laboratory tests and a plain radiographs of the chest, abdoman, pelvis and hips were unremarkable.

A psoas abscess was suspected and an urgent ultrasound scan was performed, as a computed tomography (CT) scan was not available due to other concurrent emergencies. Ultrasound scan revealed a large psoas abscess that had spread beyond the inguinal ligament, with marked compression of the femoral vein. A doppler imaging study of the lower limb was performed and did not reveal a deep venous thrombosis. The patient was commenced on intravenous antibiotics (co-amoxiclav, 1.2g, q.i.d.) and was taken to the operating theatre immediately for open drainage. An oblique incision was made in the right iliac fossa and a muscle splitting manoeuvre was performed in order to access the retroperitoneal space (Figure 2). A large pus collection was found (approximately two litres) and this was drained immediately (Figure 3). The wound was thoroughly irrigated and a drain was left in situ (Figures $4,5)$. The patient made an uneventful recovery and was discharged on day 5. Subsequently, he was tested positive for HIV and was commenced antiretroviral treatment.

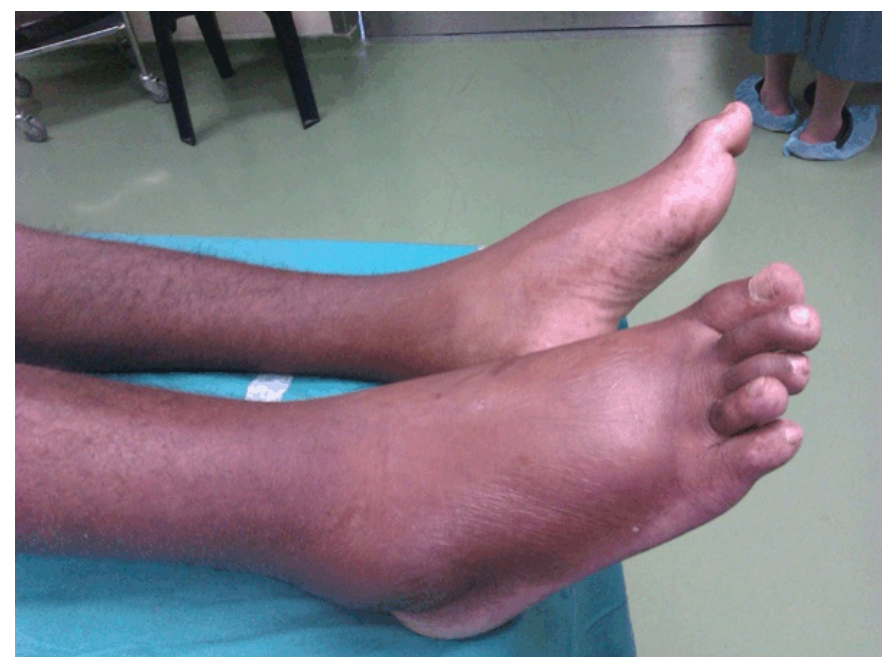

Figure 1: Patient presented with swelling of right foot extending to the calf.

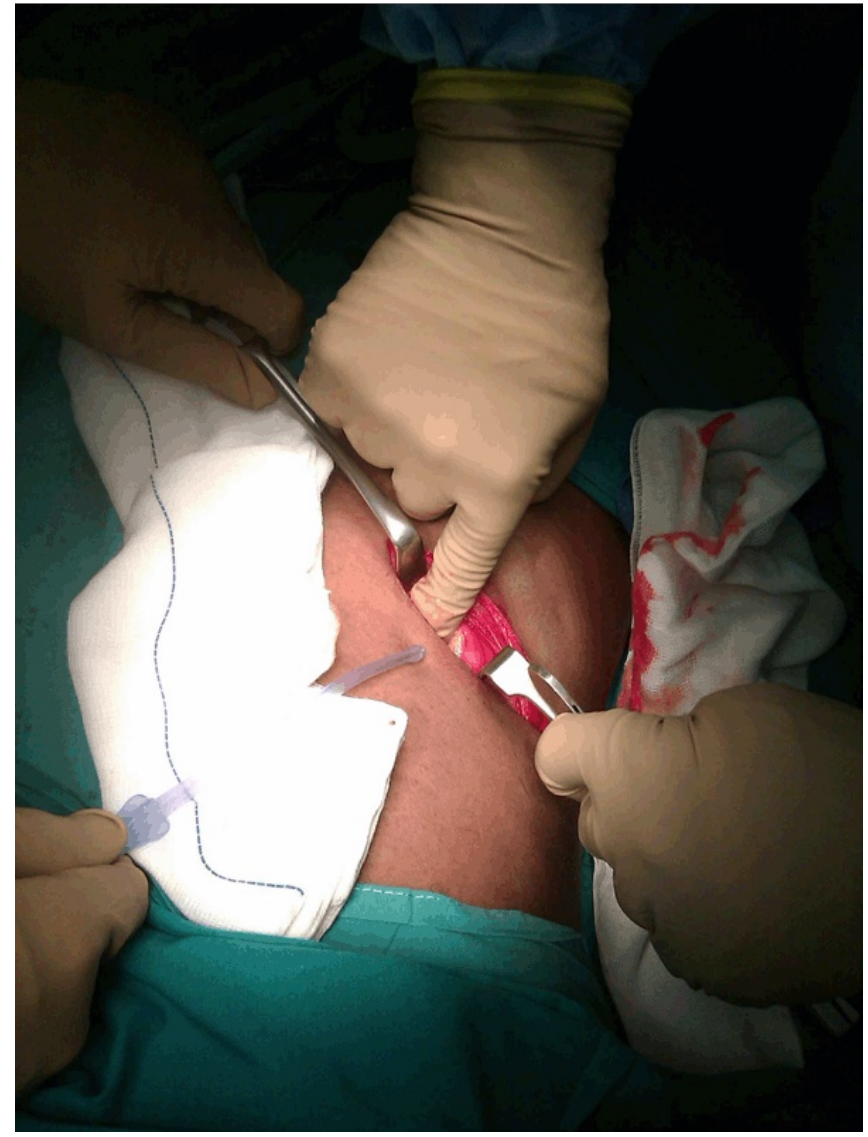

Figure 2: Dissection to access the retroperitoneal space.

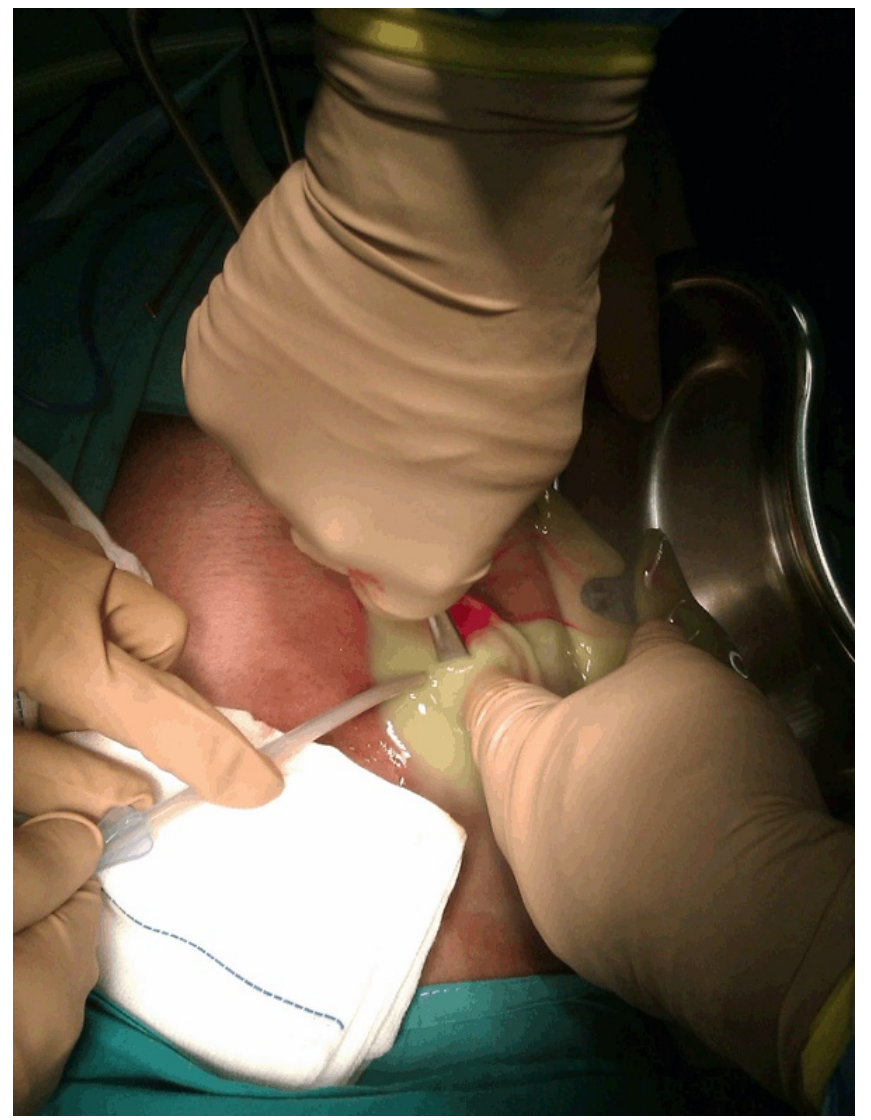

Figure 3: Retroperitoneal space showed, large gush of thick pus collection. 


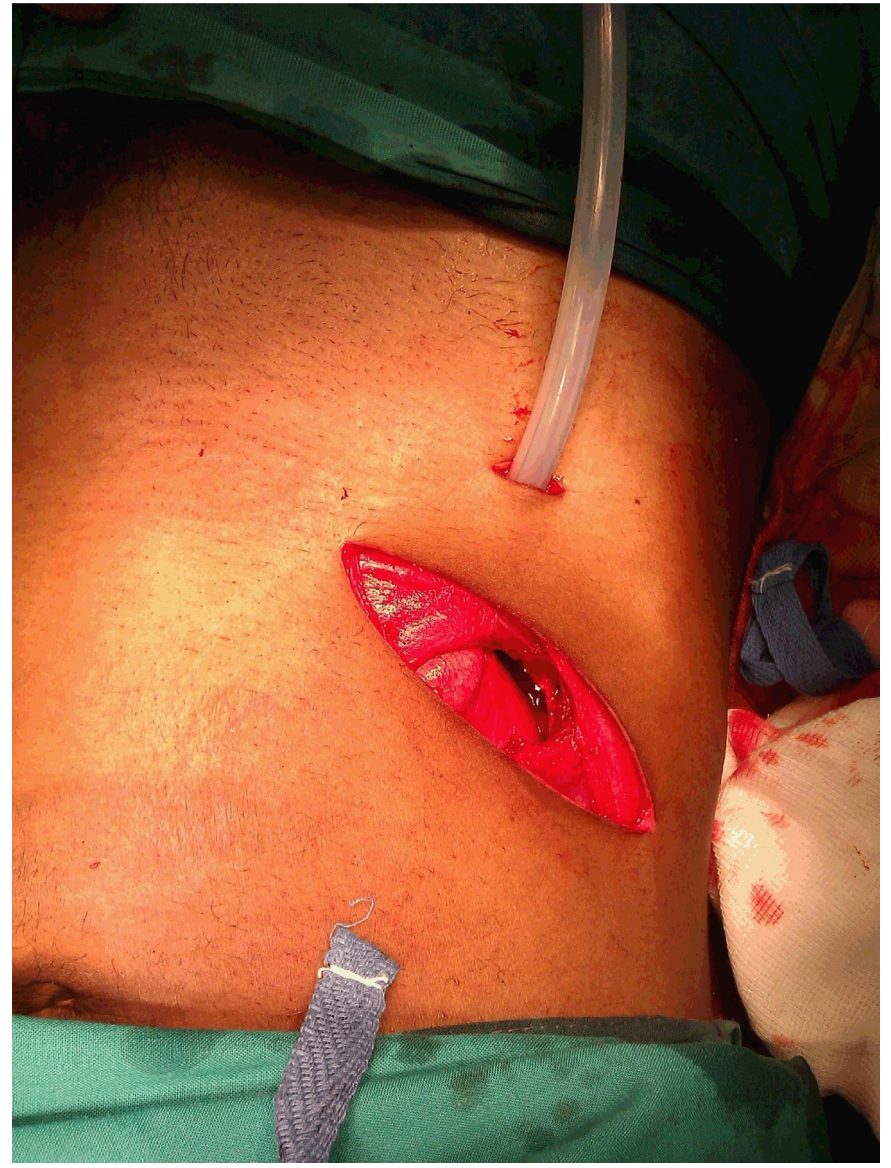

Figure 4: Drain left in situ.

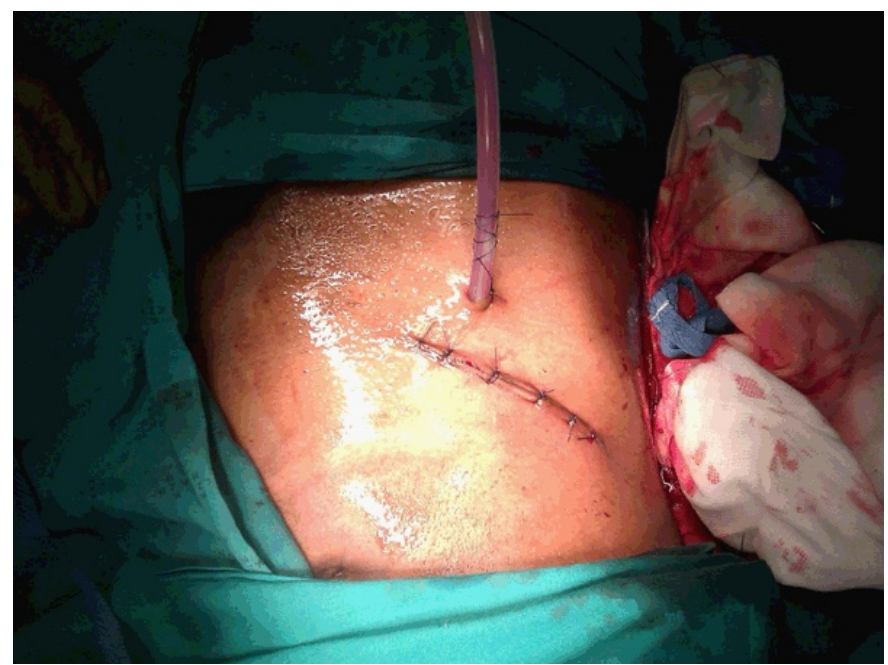

Figure 5: Wound apposed with drain in situ.

\section{DISCUSSION}

Psoas abscess was first described by Mynter et. al. in 1881 and was originally referred to as 'psoitis' [1]. A psoas abscess is a relatively rare condition and has a worldwide incidence of approximately 12 cases per
100,000 per year [2]. It carries a morality rate of up to $20 \%$ [3]. A psoas abscess is usually classified as either primary, if there is no obvious source of infection, or as secondary, if an obvious source of infection is present (such as Crohn's disease, perforated appendix etc). There is a worldwide variation in its etiology. For example, in Africa, 99.5\% of all psoas abscesses are primary, compared with only $61 \%$ in the United States and $18.7 \%$ in Europe [4]. In over $88 \%$ of primary psoas abscess cases, Staphylococcus aureus is the causative organism. However, in the developing world, Mycobacterium tuberculosis is a common cause of psoas abscess, especially in cases of to tuberculosis of the spine [4].

This case highlighted several important issues. First, the patient obviously presented with extremely vague symptoms that were not suggestive of any particular condition. It is well known that clinical presentation of psoas abscesses is often vague and non-specific (especially in children), and this can lead to a delay in diagnosis and treatment [5-7]. The classic triad of fever, back pain and limp is only present in less than $30 \%$ cases [8], and there is no single clinical sign that is specifically diagnostic of this condition. In this particular case, it could be argued that the initial diagnosis of a deep venous thrombosis was reasonable, but the diagnosis could not be realistically supported because of a lack of adequate imaging equipment at the rural hospital. In the absence of a diagnostic imaging, there was a failure to consider other possible different diagnoses, and this resulted in inappropriate management and subsequent delay in referral to our surgical unit, which was potentially disastrous.

Patients in developing countries, especially in rural areas, often have difficulty in accessing even the most basic healthcare facilities. In this case, a lack of transportation alone meant that the patient was not able to attend the follow up appointment, and this delayed the referral for another two weeks. The patient somehow managed to return at a later date and at this point a swift referral was made to our unit. It is important that clinicians working in rural settings in the developing world remain vigilant and open to consideration of this important diagnosis. A correct diagnosis continues to hinge on a high index of suspicion, with judicious use of imagining (where available). Early referral for surgical opinion and early drainage remains the cornerstone of good management that will optimise the outcome for these patients.

\section{CONCLUSION}

Psoas abscess is uncommon and its diagnosis remains challenging. Clinicians must always remain open to consideration of this diagnosis. Early referral, judicious use of appropriate imaging and early drainage remains the key to optimise patients' outcome. 


\section{Author Contributions}

Victor Kong - Substantial contributions to conception and design, Acquisition of data, Analysis and interpretation of data, Drafting the article, Revising it critically for important intellectual content, Final approval of the version to be published

George Oosthuizen - Analysis and interpretation of data, Revising it critically for important intellectual content, Final approval of the version to be published Musa Mthethwa - Analysis and interpretation of data, Revising it critically for important intellectual content, Final approval of the version to be published

Kriban Reddy - Analysis and interpretation of data, Revising it critically for important intellectual content, Final approval of the version to be published

Damian Clarke - Analysis and interpretation of data, Revising it critically for important intellectual content, Final approval of the version to be published

\section{Guarantor}

The corresponding author is the guarantor of submission.

\section{Conflict of Interest}

Authors declare no conflict of interest.

\section{Copyright}

(C) Victor Kong et al. 2013; This article is distributed under the terms of Creative Commons Attribution 3.0 License which permits unrestricted use, distribution and reproduction in any means provided the original authors and original publisher are properly credited. (Please see www.ijcasereportsandimages.com /copyright-policy.php for more information.)

\section{REFERENCES}

1. Mynter H. Acute Psoitis. Buffalo Med Surg. J 1881;21:202-10.

2. Zhou Z, Song Y, Cai Q, Zeng J. Primary psoas abscess extending to thigh adductors: case report. BMC Musculoskelet Disorder 2010;11:176.

3. Adelean MO, Taiwo SS, Onile BA. Review of psoas abscess. Afr $\mathrm{J}$ Clin Exper Microbiol 2004;5(1):55-63.

4. Ricci MA, Rose FB, Meyer KK. Pyogenic psoas abscess: worldwide variations in etiology. World $\mathrm{J}$ Surg 1986;10(5):834-3.

5. Hoffer FA, Shamberger RC, Teele RL. Ilio-psoas abscess: diagnosis and management. Pediatric Radiology 1987;17(1):23-7.

6. Isabel L, MacTaggart P, Graham A, Low B. Pyogenic psoas abscess. Australia and New Zealand Journal of Surgery 1991;61(11):857-60.

7. Oosthuizen GV, Harrower JE, Hadley GP. Psoas abscess in children: making the diagnosis. Trop Doct 2006:36(4):246-7.

8. Chern CH, Hu SC, Kao WF, Tsai J, Yen D, Lee CH. Psoas abscess: making an early diagnosis in the ED. Am J Emerg Med 1997;15(1):83-8.
Access full text article on other devices

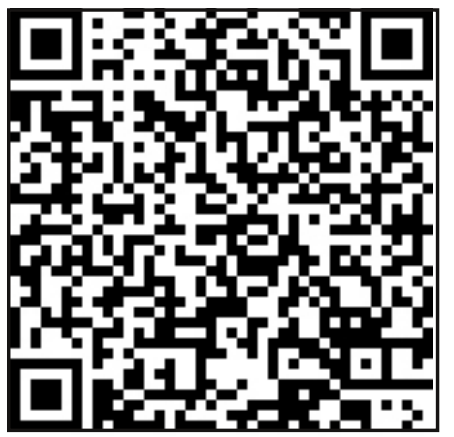

Access PDF of article on other devices

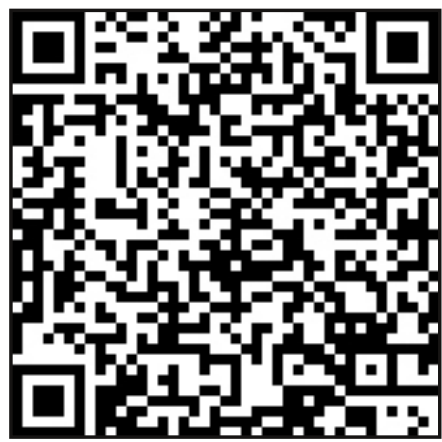

